\title{
The effects of startle and non-startle auditory stimuli on wrist flexion movement in Parkinson's disease
}

\author{
Miguel Fernández-Del-Olmo, Olalla Bello, Virginia López-Alonso, G. Márquez, José \\ A. Sánchez, Luis Morenilla, Josep Valls-Solé
}

\begin{abstract}
Startle stimuli lead to shorter reaction times in control subjects and Parkinson's disease (PD) patients. However, nonstartle stimuli also enhance movement initiation in PD. We wanted to examine whether a startle-triggered movement would retain similar kinematic and EMG-related characteristics compared to one induced by a non-startle external cue in PD patients. In this study we investigated the electromyography pattern and the reaction time during a wrist flexion movement in response to three different stimuli: a visual imperative stimulus; visual stimulus simultaneous with a non-startle auditory stimulus and with a startle auditory stimulus. Ten PD patients and ten aged matched controls participated in this study. The reaction times were faster for startle and non-startle stimuli in comparison with the visual imperative stimulus, in both patients and control subjects. The startle cue induced a faster reaction than the non-startle cue. The electromyography pattern remained unchanged across the conditions. The results suggest that the startle reaction effect for upper limb movements are unimpaired in PD patients and has different characteristics than the effect of non-startle stimuli.
\end{abstract}

Keywords

Parkinson; Wrist flexion; Intersensory facilitation; Startle

\section{Introduction}

The startle reaction is a reflex reaction generated in the brainstem in response to unexpected stimuli of various modalities, and most commonly to loud acoustic stimuli [7]. The startle reaction is characterized by a symmetrical central to peripheral activation pattern of muscles [2] and [7], where the activity of the sternocleidomastoids is the most reliable indicator of the startle reaction [2].

Previous studies have shown that, when a startling auditory stimulus is applied together with a visual "go" signal in order to initiate a task, healthy subjects shorten dramatically their reaction time [20] and [21]. This phenomenum is called the StartReact effect [20] and [21]. The fast reaction time has been shown to be similar to that of the startle reaction itself [4], suggesting that the response observed could be the result of a startle reaction and superimposed voluntary components. According with this hypothesis, the form of the EMG pattern of the voluntary movement should change as a result of the startle reaction. However, a previous study showed that the shorter reaction time induced by the startle stimulus did not change the EMG pattern of ballistic flexion or extension wrist movements [21], suggesting that the motor programmes can in some circumstances be prepared in advance and triggered earlier by a startle stimulus [21].

Several studies have found reaction time speeding effects in upper limb movements, in PD, in response to startle stimuli [6] and [19]. However, it is unclear whether this reaction time facilitation is due to simply the addition of an acoustic cue to a visual IS or whether additional facilitation is provided by a loud and startling acoustic cue. For instance, it has been reported that non-startle external cues also facilitate movement initiation in PD [1], [8], [9] and [15], suggesting that PD patients rely more on external cues to initiate their movements than control subjects. Therefore, the StartReact effect reported in PD patients could also be the result of the specific facilitator effect induced by the external cue rather than the startle reaction itself. In addition, no previous studies have explored whether a startle-triggered movement would retain similar kinematic and EMG-related characteristics compared to one induced by a non-startle external cue in PD patients. To this end we studied the effects of both startle and non-startle auditory stimuli on a ballistic wrist flexion movement in PD patients and control subjects. 


\section{Methods and subjects}

Ten subjects affected by PD (mean age $57 \pm 9$ years) were included in our study. A second group of ten age and gender-matched healthy subjects (mean age of $56 \pm 11$ years) with no history of neurologic disease participated in this study as controls. No subject showed dementia as assessed by mini-mental state examination (MMSE). All tests were carried out while the patients were ON medication, confirmed by a neurologist. Details of the subjects are shown in Table 1 . The study was approved by the local ethics committee. All participants provided informed consent (see Table 2).

Table 1. Details of Parkinson's disease patient's characteristics.

\begin{tabular}{lccccc}
\hline $\begin{array}{l}\text { Patient } \\
\text { number }\end{array}$ & $\begin{array}{c}\text { Age } \\
\text { (years) }\end{array}$ & Sex & $\begin{array}{c}\text { Disease } \\
\text { duration } \\
\text { (years) }\end{array}$
\end{tabular} Type H\&Y $\quad$ UPDRS motor $\quad$ Medication per day (mg)

\begin{tabular}{|c|c|c|c|c|c|c|c|}
\hline 1 & 67 & M & 3 & $\mathrm{M}$ & 2 & 15 & $\begin{array}{l}\text { Levodopa/Benserazide 500/125, Rasagiline } 1 \text {, } \\
\text { Pramipexole } 2.64\end{array}$ \\
\hline 2 & 66 & $\mathrm{~F}$ & 12 & $\mathrm{~T}$ & 2.5 & 12 & $\begin{array}{l}\text { Levodopa/Carbidopa 200/50, } \\
\text { Levodopa/Benserazide 550/137.5, Rotigotine 6, } \\
\text { Rasagiline 1, Amantadine } 200\end{array}$ \\
\hline 3 & 58 & $\mathrm{M}$ & 6 & $\mathrm{M}$ & 2 & 23 & $\begin{array}{l}\text { Levodopa/Carbidopa 500/125, Rasagiline 1, } \\
\text { Rotigotine } 4\end{array}$ \\
\hline 4 & 60 & $\mathrm{M}$ & 6 & A & 2 & 12 & $\begin{array}{l}\text { Levodopa/Carbidopa 800/200, Entacapone } 800 \text {, } \\
\text { Pramipexole } 3.15\end{array}$ \\
\hline 5 & 60 & M & 7 & M & 2 & 13 & $\begin{array}{l}\text { Levodopa/Carbidopa 500/125, Ropinirole 12, } \\
\text { Trihexyphenidyl } 2\end{array}$ \\
\hline 6 & 62 & $\mathrm{~F}$ & 2 & $\mathrm{AR}$ & 3 & 31 & $\begin{array}{l}\text { Levodopa/Carbidopa 600/150, Entacapone 600, } \\
\text { Rotigotine 6, Pramipexole 3.15 }\end{array}$ \\
\hline 7 & 68 & $\mathrm{M}$ & 1 & AR & 2.5 & 16 & $\begin{array}{l}\text { Levodopa/Carbidopa 150/37.5, Entacapone } 600 \text {, } \\
\text { Rasagiline } 1\end{array}$ \\
\hline 8 & 50 & M & 5 & $\mathrm{AR}$ & 2 & 19 & $\begin{array}{l}\text { Levodopa/Carbidopa 300/75, Rasagiline } 1 \text {, } \\
\text { Rotigotine } 8\end{array}$ \\
\hline 9 & 39 & $\mathrm{~F}$ & 1 & $\mathrm{~T}$ & 2 & 18 & $\begin{array}{l}\text { Levodopa/Carbidopa 375/93.75, Entacapone 600, } \\
\text { Rasagiline } 1\end{array}$ \\
\hline 10 & 45 & $\mathrm{M}$ & 3 & $\mathrm{M}$ & 2 & 11 & $\begin{array}{l}\text { Levodopa/Carbidopa 225/56.25, Entacapone } 600 \text {, } \\
\text { Rasagiline 1, Rotigotine } 4\end{array}$ \\
\hline Mean & 57 & & 4.6 & & 2.2 & 17 & \\
\hline SD & 9 & & 3.37 & & 0.34 & 6.8 & \\
\hline
\end{tabular}

Abbreviations: AR, akinetic-rigid; T, tremor-dominant; M, mixed; H\&Y, Hoehn and Yard Scale; UPDRS, Unified Parkinson Disease Rating Scale.

Table 2. Reaction time, onset latency and duration of the EMG bursts.

\begin{tabular}{|c|c|c|c|c|c|c|}
\hline \multirow{2}{*}{$\begin{array}{l}\text { Condition } \\
\text { Parameters/group }\end{array}$} & \multicolumn{2}{|c|}{ IS } & \multicolumn{2}{|c|}{$\mathrm{IS}+\mathrm{AS}$} & \multicolumn{2}{|c|}{$\mathrm{IS}+\mathrm{SS}$} \\
\hline & PD & Control & $\mathrm{PD}$ & Control & PD & Control \\
\hline \multicolumn{7}{|l|}{ Wrist flexion } \\
\hline Reaction time (ms) & $342 \pm 14$ & $313 \pm 13$ & $287 \pm 10$ & $275 \pm 11$ & $240 \pm 18$ & $200 \pm 18$ \\
\hline Onset Ag1 (ms) & $272 \pm 56$ & $258 \pm 54$ & $204 \pm 46$ & $231 \pm 29$ & $131 \pm 44$ & $126 \pm 19$ \\
\hline Duration Ag1 (ms) & $113 \pm 60$ & $128 \pm 71$ & $118 \pm 58$ & $125 \pm 83$ & $114 \pm 63$ & $118 \pm 67$ \\
\hline Time Ag1-Ag2 (ms) & $155 \pm 61$ & $180 \pm 87$ & $163 \pm 50$ & $175 \pm 101$ & $145 \pm 66$ & $163 \pm 74$ \\
\hline Time Ag1-Ant (ms) & $35 \pm 17$ & $50 \pm 14$ & $36 \pm 22$ & $44 \pm 15$ & $37 \pm 20$ & $39 \pm 15$ \\
\hline Duration Ant (ms) & $89 \pm 41$ & $127 \pm 47$ & $90 \pm 54$ & $121 \pm 49$ & $77 \pm 49$ & $106 \pm 72$ \\
\hline Duration Ag2 (ms) & $133 \pm 42$ & $127 \pm 17$ & $141 \pm 37$ & $139 \pm 16$ & $139 \pm 54$ & $130 \pm 14$ \\
\hline
\end{tabular}




\subsection{Procedure}

Subjects were asked to initiate a ballistic wrist flexion in response to a visual imperative stimulus (IS), a white $25 \mathrm{~cm}^{2}$ square displayed on a black computer screen situated in front of the subject. A warning auditory cue preceded the IS with a variable period of 3-5 s. In some trials the onset of the IS was simultaneous with an auditory stimulus (AS) of a $750 \mathrm{~Hz}$ tone burst lasting $30 \mathrm{~ms}$ with intensity of $80 \mathrm{~dB}$, while in other trials the onset of IS was simultaneous with a startling auditory stimulus (SS) of the same tone but with an intensity of $130 \mathrm{~dB}$. The intensity of the sound was measured at $1 \mathrm{~m}$ distance from the sound source. We measured the stimulus intensity of each auditory stimulus type, using a type 2204 Bruel and Kjaer Impulse Precision Sound Level Meter.

The experimental session consisted of one block of 25 trials: 15 trials consisted of only the IS, 5 trials of the IS and an AS (IS + AS) and 5 trials with the IS and a SS (IS + SS). The order of the trials was semi-randomized to avoid two or more consecutives IS + SS trials. The inter-trial interval of varied between 10 and $15 \mathrm{~s}$. The subjects were instructed to concentrate on responding to the IS, regardless of the presence of the AS or SS. Before beginning with the experiment subjects performed 15 trials in order to minimize the variability of the performance. During the practice of the wrist movement the subjects trained to achieve a $50^{\circ}$ wrist flexion, using an oscilloscope to provide feedback. After the practical trials, the oscilloscope was removed to avoid distraction of the visual stimulus displayed on the computer screen. No feedback of their performance was provided to the subjects during the testing trials.

\subsection{Wrist flexion movement}

The subjects were sitting on a comfortable chair besides a table, with their forearm and hand enclosed in a metallic device made of two parts. The part containing the subject's forearm was fixed to the surface of the table, while the part holding the subject's hand could freely move within a range of -90 to +90 deg with respect to the fixed part, which was connected to a potentiometer. The subject's forearm and hand were fully supported halfway between pronation and supination of the elbow, such that only movements of the wrist joint were allowed. The subjects were instructed to react by making a brisk self-terminated $50^{\circ}$ flexion of the wrist in response to an imperative stimulus.

\subsection{Data analysis}

Electromyographic activity (EMG) was recorded from flexor carpial radialis (wrist flexor muscle, WF) and extensor carpial radialis (wrist extensor muscle, WE) muscles. EMG was recorded with pairs of surface silver/silver chloride electrodes $(0.7 \mathrm{~cm}$ diameter $)$ placed $2-3 \mathrm{~cm}$ apart, straddling the motor point, on the belly of flexor carpial radialis and over the belly of the flexor carpial radialis. A ground electrode was attached to the muscle insertion on the anterior side of the wrist. In addition, EMG from the right and left sternocleidomastoids was recorded during the practice trials to ensure that the startle reflex only occurred with the startle stimulus. To reduce movement artefacts, the electrodes were taped firmly in place and a bandage was applied to the limb to avoid cable movements. The raw EMG signals were amplified and filtered with a band-pass filter of $30 \mathrm{H}-1 \mathrm{kHz}$ (Digitimer Ltd.). Signals were digitized at $2 \mathrm{kHz}$ (CED Power1401, Cambridge Electronic Design, Cambridge, UK) and stored for off-line analysis.

The potentiometer connected to the fixed part of the metallic device in which the hand was positioned was integrated with the CED Power1401 amplifier to record the hand displacement in synchronization with the EMG recordings. The frequency sample was $1000 \mathrm{~Hz}$.

The onset latency and offset of EMG activity were visually determined using an interactive cursor of $1 \mathrm{~ms}$ resolution. Reaction time was measured as the time between the imperative stimulus and the start of the hand flexion movement. The start of the hand flexion movement was calculated using a rising threshold method (Signal software, Cambridge Electronic Design Ltd.) The rising method is an automatic procedure of the Signal software. This method calculates the point at which the data rise above a threshold level that is greater than the noise rejection (noise rejection is evaluated as the value for which the data must rise before a peak is accepted as a peak). Endpoint of movement was visually determined at the time of the peak displacement. Movement duration was calculated as the time from the start of hand flexion movement until the endpoint.

\subsection{Statistical analysis}

Repeated-measures analysis of variance (ANOVA) was performed with condition (IS, IS + AS, IS + SS) and group (control, PD) as factors for the following variables: reaction time, the onset latency 
and duration of first WF activation (Ag1), duration of second WF activation (Ag2), duration of WE activation (Ant), time between the first and second WF activation (time Ag1-Ag2) and time Ag1-Ant. A post-hoc analysis (Sidak) was carried out whenever differences were detected with ANOVA.

All statistical analyses were performed using SPSS (SPSS, Chicago, IL). None of the data violated the normality assumption necessary to conduct parametric statistical tests. A $p$ value $\leq 0.05$ was considered statistically significant.

\section{Results}

Fig. 1A shows the mean values for the EMG activity and reaction time measured for wrist flexion in each of the conditions for control subjects and patients. The triphasic pattern characteristic of the ballistic wrist flexor movement was similar in both PD and control groups and remained unchanged across conditions. No significant effect of condition was found in the Ag1 duration, Ag2 duration, Ag1-Ag2 time, Ag1-Ant time and Ant duration. The ANOVA of the Ag1 onset latency showed a significant effect of condition $\left(F_{2,36}=42.31 p<0.0001\right.$, effect size $(E Z)=0.76$ and observed power $\left.(\mathrm{OP})=100 \%\right)$ without significant effect of group or condition $\times$ group interaction. Post hoc analysis revealed significant differences between all conditions with the fastest Ag1 onset time corresponding to the IS + SS condition and slowest to the IS condition (IS vs. IS + AS, $p=0.045$; IS vs. IS + SS, $p<0.0001$; IS + AS vs. IS +SS, $p<0.0001)$.

A

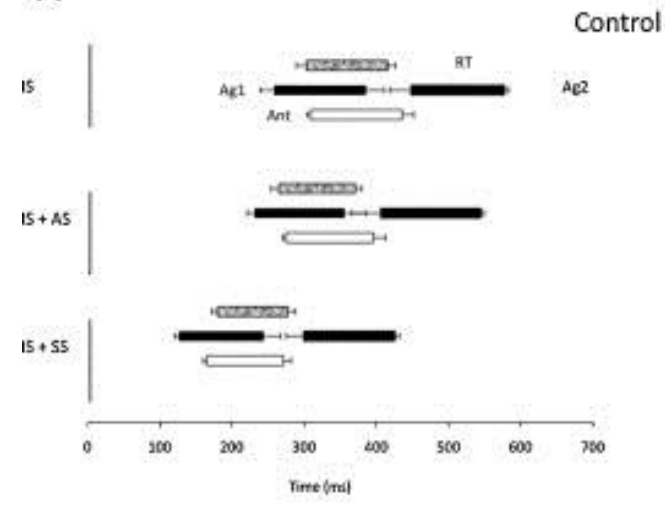

PD
B

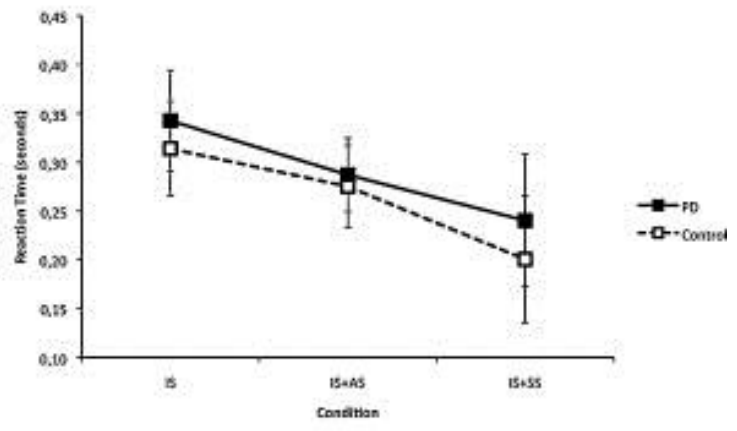

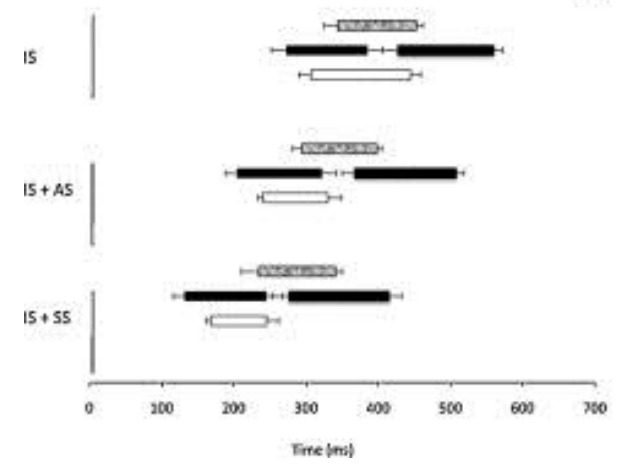

Fig. 1. (A) Schematic representation of the mean EMG pattern during wrist flexion in controls and PD patients across conditions. (IS) imperative visual stimulus; (IS + AS) visual plus auditory stimulus; (IS + SS) visual plus startle auditory stimulus; (RT) reaction time for the onset movement; (Ag1) first agonist burst; (Ag2) second agonist burst (Ant) antagonist burst. The leftward extent of the RT bar represents the reaction time, the length of the bar the movement duration, and the whisker at the right side of the standard error of the mean, movement duration. The leftward extent of the Ag1, Ag2 and Ant bars represents the mean onset latency and the length of the bars represents the duration of the EMG bursts. The whisker at the left of each bar represents the standard error of the mean onset latency while the whisker at the right side of the bar represents the standard error of the mean duration of the EMG bursts. Note how in controls and PD patients the pattern of the EMG bursts (i.e. the interburst interval and the burst durations) is the same across conditions. (B) Reaction times for wrist flexion movement across conditions. *Indicate significant differences between conditions $(p<0.05)$. 
The ANOVA of the reaction time showed a significant main effect for condition $\left(F_{2,36}=51.24\right.$ $p<0.0001, \mathrm{EZ}=0.82$, $\mathrm{OP}=100 \%$ ) but no significant effect for group nor a condition $\times$ group interaction. Both PD patients and controls decreased significantly their reaction time in the IS + AS condition in comparison with the IS condition $(p<0.0001)$ and this reduction in reaction time was more pronounced in the presence of the startle stimulus (IS + SS vs. IS + AS $p<0.0001$; IS + SS vs. IS $p<0.0001)$. Fig. 1B summarizes the main results in regard to the statistical analysis of reaction time values for control subjects and patients in the three experimental conditions.

\section{Discussion}

The main finding of the study is that the effects of the startling stimulus are similar in PD patients and healthy subjects during a ballistic wrist flexion. In addition, the startle cue induced a faster reaction compared with the non-startle cue. These observations suggest that the StartReact effect for upper limb movements in PD patients is unaffected and different from the response to a non-startle stimulus.

\subsection{Wrist flexion initiation in response to auditory (non-startle) stimulus}

When a non-startle auditory stimulus was presented simultaneously with the IS, our PD patients were able to speed up the wrist flexion movement in comparison with the presentation of just the IS. The mechanism underlying this effect remains unclear, although non dopaminergic pathways such as the cerebello-thalamocortical pathways have been implicated [10]. However, our findings suggest that the beneficial effect of sensory stimuli is not specific to PD, since both PD and control subjects demonstrated similar magnitudes of reaction time facilitation in onset of wrist movement in response to the auditory stimulus. This general beneficial effect could be attributed to intersensory facilitation, which is a phenomenon in which the reaction time to a stimulus in one sensory modality is shortened significantly if the reaction stimulus is paired with a stimulus in another modality (an accessory stimulus) that is presented in close temporal proximity [16]. Our findings are in line with another study showing that intersensory facilitation is of equal magnitude in PD and control subjects [17].

\subsection{Wrist flexion initiation in response to auditory startle stimulus}

We replicated findings showing that control subjects and PD patients speeded up wrist flexion movement in response to the combination of a startling stimulus and the imperative cue [19] and [21]. Previous studies have reported that the startle reaction is characterized by flexion actions [14] and [23], which could suggest that in our task the fastest reaction time is the result of a voluntary movement superimposed on the startle reaction. However, the motor program remains undisturbed since no differences were found in the EMG pattern, which is in agreement with previous studies [19] and [21]. This pattern is not simply related to reflex mechanisms using peripheral feedback as a result of movement, but rather seems to be preprogrammed in the central nervous system [13], as it also occurs in deafferented subjects [11] and [18]. Although, several hypothesis have been suggested to explain the StartReact effect [5] and [21] the mechanism of reaction time facilitation by startle is still unclear.

In the present study we also demonstrated the wrist movement responses to both auditory and startle stimuli were similar in controls and PD patients. In both groups reaction time were shorter in trials containing the SS than in trials of the other two conditions. A "stimulus-intensity effect", i.e. a decrease in reaction time with an increase in the intensity of a stimulus [12] may explain the decrease in reaction time between the two auditory conditions. However, the magnitude of the reaction time reduction we observed in both groups is larger than what could be expected for the increase in stimulus intensity. This is in line with the observations of Carlsen and colleagues [3], who reported that startle produces early response latencies that are distinct from stimulus intensity.

Our study has two important limitations. First, the EMG activation of the sternocleidomastoids was not recorded during the testing session. It has been previously shown that the response of the sternocleidomastoids to a startle sound is significantly delayed in PD patients [22]. A dissociation between the onsets of the sternocleidomastoids and the movement during the startle trials could help to a better understanding of the mechanism involved in each response. Second, our PD patients were tested in ON condition. Although, it has been reported that the pathways involved in the startle reaction are not under dopaminergic control [22] we cannot ascertain whether our findings indicate a lack of impairment or that the impairment was restored by dopamine.

In summary, both controls and PD patients are able to reduce their reaction time of flexion wrist movements in response to a non-startle and startle auditory stimuli. The startle cue induced a faster 
reaction compared with the non-startle cue. This is of relevance since it suggests that StartReact effect for upper limb movements are unimpaired in PD patients and has different characteristics than the effect of non-startle stimuli.

\section{Funding sources}

This work was supported by Ministerio de Ciencia e Innovación(DEP2011-22466), Spain.

\section{Acknowledgment}

To Noa Fogelson for reviewing this manuscript.

\section{References}

[1] A. Burleigh-Jacobs, F.B. Horak, J.G. Nutt, J.A. Obeso Step initiation in Parkinson's disease: influence of levodopa and external sensory triggers Mov. Disord., 12 (1997), pp. 206-215

[2] P. Brown, J.C. Rothwell, P.D. Thompson, T.C. Britton, B.L. Day, C.D. Marsden New observations on the normal auditory startle reflex in man Brain, 114 (1991), pp. 1891-1902

[3] A.N. Carlsen, C. Dakin, R. Chua, I.M. Franks Startle produces early response latencies that are distinct from stimulus intensity effects Exp. Brain Res., 176 (2007), pp. 199-205

[4] A.N. Carlsen, D. Maslovat, M.Y. Lam, R. Chua, I.M. Franks Considerations for the use of a startling acoustic stimulus in studies of motor preparation in humans Neurosci Biobehav. Rev., 35 (2011), pp. 366-376

[5] A.N. Carlsen, D.I.M. Maslovat Franks Preparation for voluntary movement in healthy and clinical populations: evidence from startle Clin. Neurophysiol., 123 (2012), pp. 21-33

[6] A.N. Carlsen, Q.J. Almeida, I.M. Franks Using a startling acoustic stimulus to investigate underlying mechanisms of bradykinesia in Parkinson's disease Neuropsychologia, 51 (2013), pp. 392-399

[7] M. Davis, D.S. Gendelman, M.D. Tischler, P.M. Gendelman A primary acoustic startle circuit: lesion and stimulation studies J. Neurosci., 2 (1982), pp. 791-805

[8] L.E. Dibble, D.E. Nicholson, B. Shultz, B.A. MacWilliams, R.L. Marcus, C. Moncur Sensory cueing effects on maximal speed gait initiation in persons with Parkinson's disease and healthy elders Gait Posture, 19 (2004), pp. 215-225

[9] M. Fernández-Del-Olmo, O. Bello, V. Lopez-Alonso, J. Andrés Sanchez, D. Santos-García, J. Valls-Solé The effects of auditory startle and non-startle stimuli on step initiation in Parkinson's disease Mov. Disord., 27 (2012), pp. $1570-1573$

[10] M. Glickstein, J. Stein Paradoxical movement in Parkinson's disease Trends Neurosci., 14 (1991), pp. 480-482

[11] M. Hallett, B.T. Shahani, R.R. Young EMG analysis of stereotyped voluntary movements in man J. Neurol. Neurosurg. Psychiatry, 38 (1975), pp. 1154-1162

[12] D.L. Kohfeld Effects of the intensity of auditory and visual ready signals on simple reaction time J. Exp. Psychol., 82 (1969), pp. 88-95

[13] B. Köster, G. Deuschl, M. Lauk, J. Timmer, B. Guschlbauer, C.H. Lücking Essential tremor and cerebellar dysfunction: abnormal ballistic movements J. Neurol. Neurosurg. Psychiatry, 73 (2002), pp. 400-405

[14] C. Landis, W.A. Hunt The Startle Pattern Farrar \& Rinehart, New York (1939)

[15] E.B. Montgomery Jr., J. Nuessen, D.S. Gorman Reaction time and movement velocity abnormalities in Parkinson's disease under different task conditions Neurology, 41 (1991), pp. 1476-1481

[16] R.S. Nickerso Intersensory facilitation of reaction time: energy summation or preparation enhancement? Psychol. Rev., 80 (1973), pp. 489-509

[17] F.M. Plat, P. Praamstra, M.W. Horstink Redundant-signals effects on reaction time, response force, and movement-related potentials in Parkinson's disease Exp. Brain Res., 130 (2000), pp. 533-539

[18] J.C. Rothwell, M.M. Traub, B.L. Day, J.A. Obeso, P.K. Thomas, C.D. Marsden Manual performance in deafferented man Brain, 105 (1982), pp. 515-542

[19] F. Valldeoriola, J. Valls-Solé, E. Tolosa, P.J. Ventura, F.A. Nobbe, M.J. Martí Effects of a startling acoustic stimulus on reaction time in different Parkinsonian syndromes Neurology, 51 (1998), pp. 1315-1320

[20] J. Valls-Solé, A. Solé, F. Valldeoriola, E. Muñoz, L.E. Gonzalez, E.S. Tolosa Reaction time and acoustic startle in normal human subjects Neurosci. Lett., 195 (1995), pp. 97-100

[21] J. Valls-Solé, J.C. Rothwell, F. Goulart, G. Cossu, E. Muñoz Patterned ballistic movements triggered by a startle in healthy humans J. Physiol., 516 (1999), pp. 931-938

[22] M. Vidailhet, J.C. Rothwell, P.D. Thompson, A.J. Lees, C.D. Marsden The auditory startle response in the Steele-Richardson-Olszewski syndrome and Parkinson's disease Brain, 115 (1992), pp. 1181-1192

[23] D.E. Wilkins, M. Hallett, M.M. Wess Audiogenic startle reflex of man and its relationship to startle syndromes. A review Brain, 109 (1986), pp. 561-573 